\title{
Neurovascular compression syndrome of the brain stem with opsoclonus-myoclonus syndrome combined with vestibular paroxysmia and autonomic symptoms
}

\author{
Yusuke Morinaga*, Kouhei Nii, Kimiya Sakamoto, Ritsurou Inoue, Takafumi Mitsutake, \\ Hayatsura Hanada
}

Department of Neurosurgery, Fukuoka University Chikushi Hospital, Chikushino-city, Fukuoka, Japan.

\begin{abstract}
Summary We describe a rare case of neurovascular compression syndrome (NVCS) of the brain stem and opsoclonus-myoclonus syndrome (OMS) complicated with vestibular paroxysmia (VP) and autonomic symptoms. Moreover, we discuss the case with respect to the available information in medical literature. A 36-year-old man with vertigo and nausea had difficulty standing, and was transported by an ambulance to our hospital. He had VP, opsoclonus, cervical myoclonus, anxiety, and restless legs syndrome. Magnetic resonance imaging at hospitalization showed that the dolichoectatic vertebral artery was in contact with the postero-lateral side of the pontomedullary junction. He was diagnosed with NVCS of the brain stem (most likely of the input to the vestibular nucleus) associated with contact with the dolichoectatic vertebral artery. Combination therapy using multiple antiepileptic drugs, such as low-dose carbamazepine, clonazepam, and lacosamide, improved his clinical symptoms. He was finally able to walk and was discharged on day 42 after admission. He is being routinely followed-up since then. Further research is needed to confirm the validity of the combination therapy.
\end{abstract}

Keywords: Neurovascular compression syndrome, brainstem, opsoclonus myoclonus syndrome, vestibular paroxysmia, autonomic symptoms

\section{Introduction}

Opsoclonus-myoclonus syndrome (OMS) (1-3) is a rare disease with opsoclonus, cerebellar ataxia, and myoclonus of the trunk, limbs, and neck as the primary symptoms. The probable underlying etiologies eliciting the symptoms include viral encephalitis, malignant tumors, metabolic disorders, and degenerative diseases. Particularly, OMS as paraneoplastic nerve syndrome (PNS), wherein, various neurological symptoms manifest because of the "remote effect" of malignant tumors, is an important etiology. Neuroblastoma is present in about half of the children diagnosed with OMS, and in adults, OMS can be seen as a complication of lung cancer, breast cancer, and ovarian cancer.

\footnotetext{
*Address correspondence to:

Dr. Yusuke Morinaga, Department of Neurosurgery and Neuroscience, Fukuoka University Chikushi Hospital, 1-1-1 Zokumyoin, Chikushino-shi, Fukuoka Prefecture 818-8502, Japan.

E-mail: yu_the_morio@yahoo.co.jp
}

While, vestibular paroxysmia (VP) may manifest when arteries in the cerebellar pontine angle cause a segmental, pressure-induced dysfunction of the eighth nerve. There are some case reports of neurovascular compression syndrome (NVCS) presenting with VP (4-7). We encountered a rare case of NVCS of the brainstem complicated with vestibular paroxysmia (VP) and autonomic symptoms combined with OMS. Here, we present the clinical course of the disease and report the findings in light of the existing scientific literature.

\section{Case Report}

A 36-year-old man with no remarkable medical history and chief complaints of vertigo and nausea, visited an ophthalmologist. Magnetic resonance imaging (MRI) showed no abnormality, and he was prescribed betahistine, kallidinogenase, adenosine triphosphate disodium hydrate granules, and alprazolam for the aforementioned symptoms. A few days later, his vertigo progressed and he was unable to walk, and 


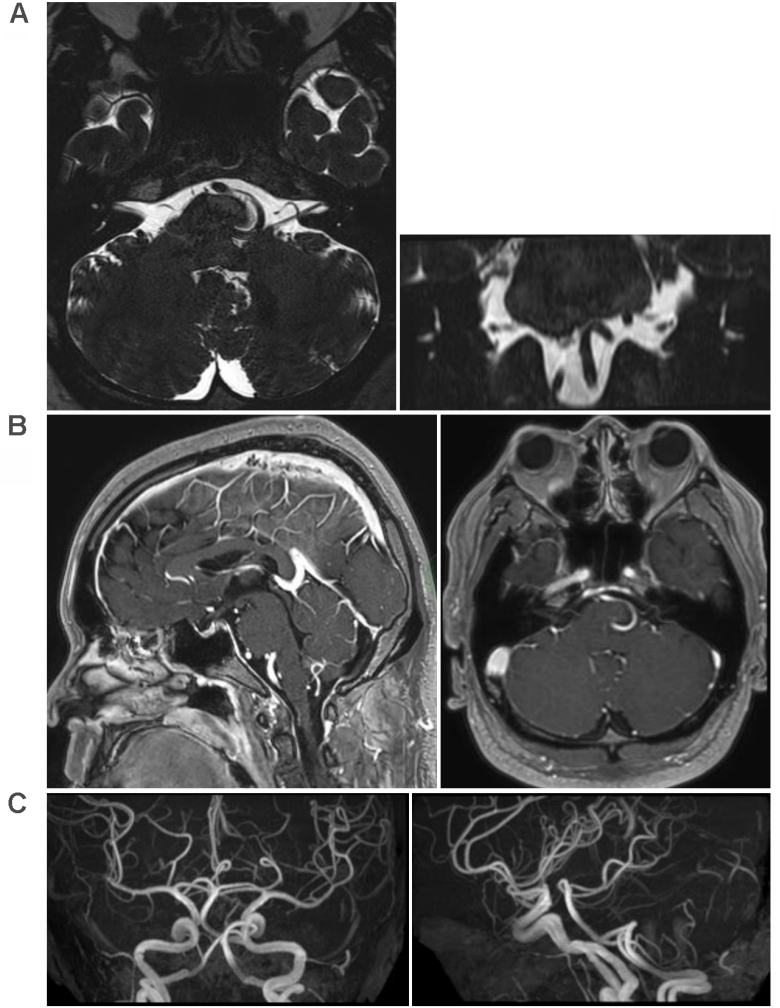

Figure 1. Head magnetic resonance images at hospitalization. A. Magnetic resonance imaging (MRI) Constructive Interference Steady State at hospitalization shows that the dolichoectatic vertebral artery is in contact with the posterior lateral side of the pontomedullary junction (left, axial view; right, coronal view). B. Gadolinium-T1weighted MRI at hospitalization shows no tumor and findings similar to Figure 1A. C. MR angiography at hospitalization shows the dolichoectatic vertebral artery.

was transported to our hospital by an ambulance. At hospitalization, he had clear consciousness, blood pressure was $110 / 62 \mathrm{mmHg}$, and body temperature was $36.2^{\circ} \mathrm{C}$. He had vertigo and nausea, but without facial nerve palsy and tinnitus. He showed no decline in hearing or hearing sensitivity, no sense of ear obstruction, and no difference in hearing ability. Spontaneous nystagmus at rest was noted (horizontal nystagmus was omnidirectional, with biphasic horizontal nystagmus at bilateral lateral gaze, horizontal rotational nystagmus at upward gaze, and swaying omnidirectional saccadic abnormal eye movement during eye-opening in the dark [opsoclonus]). Marked trunk ataxia was found with no quadrupeds. $\mathrm{He}$ was unable to sit or stand. He had no other obvious neurological deficits. Momentary involuntary movements of the neck and shoulders (cervical myoclonus) were found. He also had myoclonus, restless leg syndrome, erectile dysfunction. Blood tests showed no abnormal findings (such as tumor markers) other than an LDL level of $182 \mathrm{mg} / \mathrm{dL}$.

There were no remarkable findings in the auditory brainstem response. MRI at hospitalization indicated contact of the left vertebral artery (VA) with the dorso- lateral area of the ponto-medullary junction (Figures $1 \mathrm{~A}$ and 1B). A marked bending or meandering of the vertebral artery was observed (Figure 1C). Chest-pelvic computed tomography showed no neoplastic lesion.

Based on the presented symptoms and imaging findings at admission, we suspected OMS and VP associated with NVCS caused by the left VA compressing the brainstem. Based on this initial diagnosis, the patient was administered carbamazepine (CBZ) $400 \mathrm{mg} /$ day from day 1. Although the OMS and VP persisted, the seizure frequency decreased marginally. On day 2 , rosuvastatin $5 \mathrm{mg}$ and brotizolam $0.25 \mathrm{mg}$ (oral) were started for managing the dyslipidemia and insomnia, respectively. Etizolam 1.5 $\mathrm{mg}$ /day was started on day 10. Lacosamide (LCM) 100 $\mathrm{mg}$ /day was started on day 12 which was subsequently increased to $200 \mathrm{mg} / \mathrm{day}$ on day 19 . When the frequency of the OMS symptoms and VP decreased, clonazepam (CZP) $(1.5 \mathrm{mg} /$ day $)$ was introduced to treat the restless leg syndrome. Erectile dysfunction, insomnia, and restless legs syndrome alleviated and the patient was able to walk, and was discharged on day 42 . At 12 months post discharge, his OMS occasionally presented as seizures when talking with others. He continued all the previously mentioned prescription drugs except for etizolam and brotizolam because the mental symptoms, such as insomnia and anxiety, disappeared. He has since been in the outpatient follow-up.

Written informed consent was obtained from the patient for the publication of this case report and accompanying images, and the study design was approved by the appropriate ethics review board.

\section{Discussion}

To the best of our knowledge, although there have been reports of "spino-bulbo-spinal like" reflex because of the pressure of the dolichoectatic VA on the lower brainstem (4), this is the first reported case of OMS with VP and autonomic symptoms combined with NVCS of the brainstem. The main symptoms of VP (510) usually lasts for less than one minute and occur more than 30 times a day. Spontaneous or non-rotatable vertigo attacks occur spontaneously and repeatedly over a short period. VP is often caused by direct pulsatile compression of the eighth cranial nerve by an artery at the cerebello-pontine angle.

Neurovascular compression of the eighth cranial nerve is observed in more than $95 \%$ of cases presenting VP after MRI. Particularly, the involvement of the anterior inferior cerebellar artery loop is the highest, followed by the posterior inferior cerebellar artery, vertebral artery (VA), or veins. Treatment strategies involve administration of low-dose carbamazepine (200-600 mg/day) or oxcarbazepine (300-900 mg/day), which is equally effective in children. Additionally, lamotrigine, phenytoin, gabapentin, topiramate, 
baclofen, and other non-antiepileptic drugs used for trigeminal neuralgia are recommended. However, randomized, placebo-controlled trials are currently in progress, and till date, there is no established treatment. Microvascular decompression of the eighth cranial nerve is especially applicable in cases of intractable drug resistance and non-vascular compression of the eighth cranial nerve due to tumors or cysts. Important differential diagnoses include Meniere's disease, vestibular migraine, benign paroxysmal position vertigo, epileptic vestibular seizures, paroxysmal brainstem attacks (with multiple sclerosis or stroke), nasopharyngeal hiatus, perilymphatic fistula, transient ischemic attack, and panic attack. In our case, 1) The VA was in contact with the dorsolateral surface of the ponto-medullary junction. 2) The patient presented movement disorders such as spontaneous opsoclonus, VP, ataxia, cervical myoclonus, and restless leg syndrome. 3) Moreover, the patient presented autonomic nervous system symptoms and mental symptoms, such as anxiety attack, erectile dysfunction, and insomnia, after the manifestation of the motor symptoms described previously. 4) Antiepileptic drugs targeting the sodium channels (sodium channel blockers), such as low dose CBZ and LCM, were effective. Based on the above characteristics, it is possible that NVCS in the lower brainstem, centering on the vestibular nucleus caused OMS, VP, and autonomic nervous symptoms as output system symptoms involving the vestibular nucleus.

The vestibular nucleus (11) is a nucleus where the primary afferent nerves of the vestibular neurons, derived from the vestibular organ, terminate. In addition to the primary afferent nerve, inputs from the contralateral vestibular nucleus, cerebellar cortex, cerebellar nucleus, hypoglossal nucleus, Cajal stromal nucleus, spinal cord, vestibular area of the cerebral cortex, and brainstem reticular system are also found. The targets include the motor nucleus of the extraocular muscle (articulus nucleus, trochlear nucleus, abduction nucleus) and thalamus. Additionally, nerve fiber connections in the autonomic nervous system nuclei are found. Therefore, the vestibular nucleus is more than a sensory primary relay nucleus, and transmits input from the peripheral vestibule to the center. It also receives various inputs and relays it to various parts of the central nerve and is deeply involved with motor functions, such as eye movement and maintaining the balance of the body, as well as autonomic system functions (Figure 2).

The characteristics of the neural circuit involved in the vestibular nucleus can account for all the clinical symptoms observed in our patient. We considered that the OMS was not associated with PNS because the myoclonus in the OMS was confined to the neck and shoulders, and findings suggestive of a malignant tumor were inconclusive.

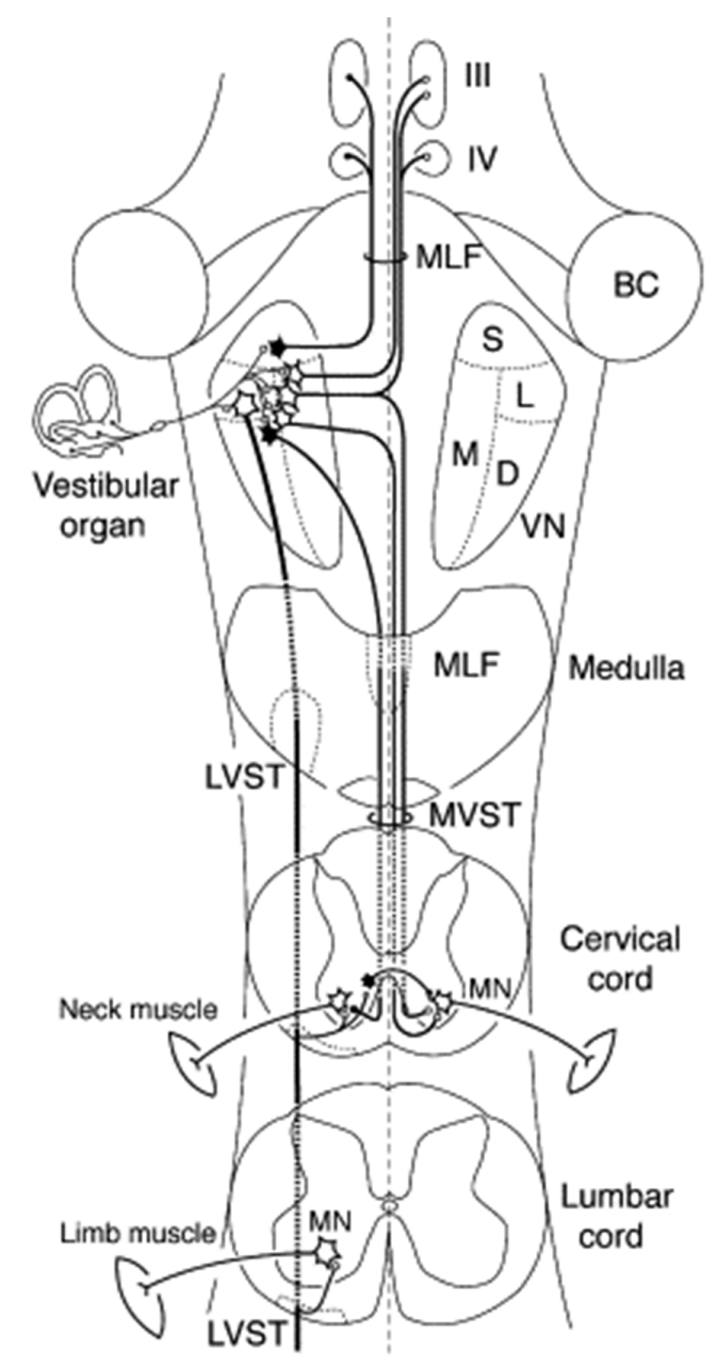

Figure 2. Neural network of the vestibular nucleus. The figure, revised partially from Shinoda et al. (11), shows the neural network of the vestibular nucleus.

Therapeutically, low dose CBZ alone did not significantly reduce seizure frequency; therefore, we added LCM, a novel sodium channel blocker, and CZP, a drug for myoclonus, restless legs syndrome, and erectile dysfunction. VP and autonomic symptoms were thought to have improved significantly. Careful followup will be continued in the future.

In conclusion, we report a rare case of NVCS of the brainstem (most likely of the input to the vestibular nucleus) with VP and autonomic symptoms combined with OMS. Low dose CBZ, LCM, and CZP combined therapy contributed to the marked decrease of seizure frequency.

\section{Acknowledgements}

We would like to thank Editage (www.editage.com) for English language editing. This study did not receive any grants from funding agencies in the public, commercial, or not-for-profit sectors. 


\section{References}

1. Honnorat J. New findings in adult opsoclonus-myoclonus syndrome. JAMA Neurol. 2016; 73:381-382.

2. Hsu SY, Young YH. Opsoclonus-myoclonus syndromereply. JAMA Otolaryngol Head Neck Surg. 2018; 144:388.

3. Kim DD, Budhram A. Opsoclonus-myoclonus syndromeadditional clinical considerations. JAMA Otolaryngol Head Neck Surg. 2018; 144:387-388.

4. Merchant SH, Vial F, Leodori G, Fahn S, Pullman SL, Hallet M. A novel exaggerated "spino-bulbo-spinal like" reflex of lower brainstem origin. Parkinsonism Relat Disord. 2018; 18:30435-30438.

5. Brandt T, Dieterich M. Vestibular paroxysmia: Vascular compression of the eighth nerve? Lancet. 1994; 343:798799.

6. Brandt T, Strupp M, Dieterich M. Vestibular paroxysmia: A treatable neurovascular cross-compression syndrome. J Neurol. 2016; 263:S90-96.
7. Hufner K, Barresi D, Glaser M, Linn J, Adrion C. Vestibular paroxysmia: Diagnostic features and medical treatment. Neurology. 2008; 71:1006-1014.

8. Lawden MC, Bronstein AM, Kennard C. Repetitive paroxysmal nystagmus and vertigo. Neurology. 1995; 45:276-280

9. Strupp M, Lopez-Escamez JA, Kim JS, Straumann D, Jen JC, Carey J, Bisdorff A, Brandt T. Vestibular paroxysmia: Diagnostic criteria. J Vestib Res. 2016; 26:409-415.

10. Strupp M, von Stuckrad-Barre S, Brandt T, Tonn JC. Teaching neuroimages: Compression of the eighth cranial nerve causes vestibular paroxysmia. Neurology. 2013; 80:77.

11. Shinoda Y, Sugiuchi Y, Izawa Y, Hata Y. Long descending motor tract axons and their control of neck and axial muscles. Prog Brain Res. 2006; 151:527-563.

(Received May 30, 2019; Revised August 13, 2019; Accepted August 22, 2019 